\title{
Electrical circuit modeling of surface structures for X-ray photoelectron spectroscopic measurements
}

\author{
T. Onur Tasci a , Ergin Atalar ${ }^{\text {a }}$, U. Korcan Demirok ${ }^{\text {b }}$, Sefik Suzer ${ }^{\text {b,* }}$ \\ ${ }^{a}$ Department of Electrical and Electronics Engineering, Bilkent University, 06800 Ankara, Turkey \\ ${ }^{\mathrm{b}}$ Department of Chemistry, Institute of Materials and Nanotechnology, Bilkent University, 06800 Ankara, Turkey
}

Received 24 June 2007; accepted for publication 17 October 2007

Available online 1 November 2007

\begin{abstract}
We model the X-ray photoelectron spectrometer and the sample with lumped electrical circuit elements, and simulate various types of conditions using a widely used computer program (PSpice) and compare the results with experimental measurements. By using the electrical model simulations, the surface voltage and the spectrum can be estimated under various types of external voltage stimuli, and the zero potential condition can be predicted accurately for obtaining a truly uncharged spectrum. Additionally, effects of several charging mechanisms (taking place during XPS measurements) on the surface potential could easily be assessed. Finally, the model enables us to find electrical properties, like resistance and capacitance of surface structures, under X-ray and low-energy electron exposure.
\end{abstract}

(C) 2007 Elsevier B.V. All rights reserved.

Keywords: X-ray photoelectron spectroscopy; Differential charging; Modeling and simulation; Silicon dioxide layers; Resistance and capacitance

\section{Introduction}

Among the various surface analytical techniques, X-ray photoelectron spectroscopy (XPS) keeps its pivotal position, due to its ability in resolving chemical identity of atoms, from measured binding energies via the photoemission process [1]. During data acquisition in XPS, a finite, measurable, and more or less steady current (0.1-20 nA) flows from the sample, which usually causes unwanted positive charging in poorly conducting samples or parts of surface heterostructures [2-5]. This charging is usually compensated by a directed flow of low-energy electrons (or ions) from an external unit (flood-gun) to the sample. Excessive flooding can also cause negative charging. This negative charging, dubbed as controlled surface charging (CSC), has successfully been utilized in deriving some chemical/physical parameters of surface structures [3-7]. Otherwise, the emphasis, until now, has mostly been on

\footnotetext{
* Corresponding author. Tel.: +90 312 2664946; fax: +90 3122664579. E-mail address: suzer@fen.bilkent.edu.tr (S. Suzer).
}

recording of the line positions (and intensities) in XPS. With the exception of a few cases [8-15], incorporation of electrical measurements for extracting additional information has been neglected.

During an XPS measurement, the total current flowing through a sample is the sum of electrons, going out of the sample (due to photoemission and secondary electrons), and into the sample (due to stray electrons or electrons from the flood-gun). This current can easily be controlled by an application of a small $(0-10 \mathrm{~V})$ external voltage, both in the form of DC and/or pulses, as it has been reported recently [12-23]. In this work, several parameters effecting charge compensation and their role(s) in determining the surface potential has been examined qualitatively, and modeled by electrical circuit elements using a readily available computer program, PSpice [24]. The precise estimation of the surface potential and the corresponding binding energy shifts have been simulated and compared with experimental measurements on a silicon substrate containing ca. $400 \mathrm{~nm}$ oxide layer. The estimation of electrical parameters using XPS measurements has been reported by various 
groups previously [12-23]. However, we believe that the use of a computer simulation program for this purpose is reported for the first time.

\section{Experimental}

A Kratos ES300 electron spectrometer with $\mathrm{Mg} \mathrm{K} \alpha \mathrm{X}$ rays (nonmonochromatic) was used for XPS measurements. Samples were electrically connected both from the top (oxide layer) and the bottom (silicon substrate) to the sample holder, which was connected to either a DC power supply or a function generator externally. Bias voltages changing from $-10 \mathrm{~V}$ to $10 \mathrm{~V}$ (with $1 \mathrm{~V}$ step) are applied to the sample holder. The current flowing through sample is measured by a multimeter (Agilent IntuiLink 34401A) connected to a personal computer. A tungsten electron gun filament is also introduced to supply low-energy electrons. For time-dependent measurements the bias is stepped and pulsed. During each pulse 200 measurements, each with a $10 \mathrm{~ms}$ window, are recorded. The voltage is stepped and pulsed for the next 200 measurements until a chosen XPS region is completed. Several scans are implemented for signal averaging [21].

\section{Results and discussion}

During XPS measurements, three types of currents flow between surroundings and the surface layer as represented in Fig. 1: (i) X-rays induce photoelectron emission current and it is represented by $I_{\mathrm{p}}$; (ii) $I_{\mathrm{s}}$, stemming from the stray electrons in the vacuum system; and (iii) $I_{\mathrm{g}}$, electrons emitted from the low-energy electron gun (a simple filament in our case). These current paths can be modeled by lumped circuit elements as also shown in Fig. 1. In the equivalent circuit model, the silicon oxide layer is represented as a parallel combination of a resistor and a capacitor. Except for the $I_{\mathrm{p}}$, the other two currents are controlled by the external

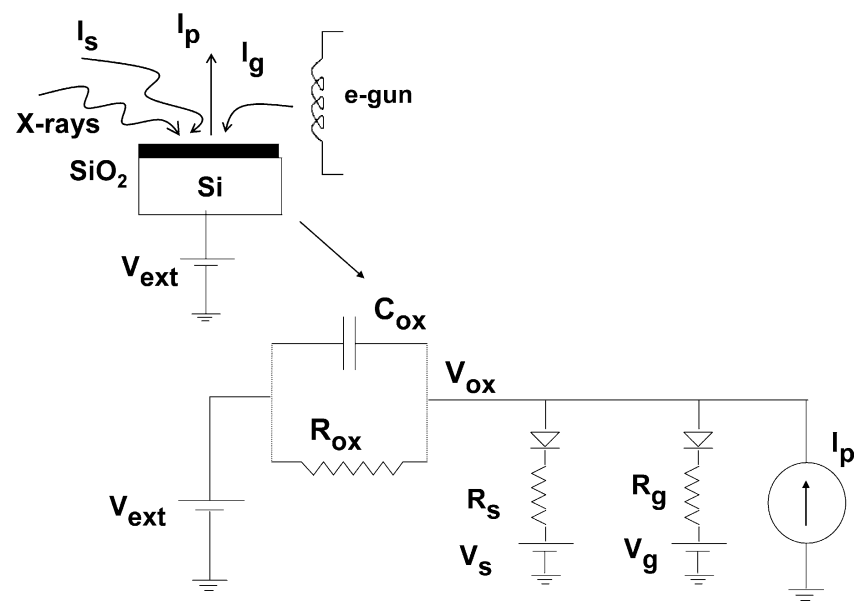

Fig. 1. Schematic representation of the various currents flowing through the sample during XPS measurements when the sample is stressed externally, and its equivalent circuit model. voltage source. Accordingly, the stray electron current path $\left(I_{\mathrm{s}}\right)$ is modeled by a series combination of an ideal diode, a resistor $\left(R_{\mathrm{s}}\right)$ and a DC voltage source $\left(V_{\mathrm{s}}\right) . V_{\mathrm{s}}$ is the minimum oxide voltage (surface voltage) to start the attraction of the stray electrons to the sample. If the surface voltage reaches a suitable value $\left(>V_{\mathrm{s}}\right)$ the diode turns on. Similarly, the electron gun current path is represented by a series connection of a diode, resistor $\left(R_{\mathrm{g}}\right)$ and another DC voltage source $\left(V_{\mathrm{g}}\right) . I_{\mathrm{p}}$ is represented in the circuit by a simple DC constant current source flowing towards the surface.

To find the various electrical parameters of this model, three types of electrical and/or XPS measurements are needed. The first measurement involves recording of the resultant total current $\left(I_{\mathrm{T}}\right)$ flowing through the sample while changing DC voltage levels from $-10 \mathrm{~V}$ to $+10 \mathrm{~V}$ (see Fig. 2a). The total current exhibits two different behaviors for the given voltage values. In Region 1, the current stays nearly constant, which corresponds to the state where the stray and gun-electrons are repelled off by the negative bias. This situation is represented in the equivalent circuit by the off-states of the two diodes. Here, the only current flowing between the surroundings and the sample is the photoelectron current $\left(I_{\mathrm{p}}\right)$. In Region 2, the current changes almost linearly with the bias voltage. This condition corresponds to the state where the electron gun and stray electron current paths are on. The second type of measurements involves recording of the binding energy spectra of the $\mathrm{Si}^{4+} 2 \mathrm{p}$. This corresponds to the two arbitrarily chosen points in Region 2 (for example, at $+2 \mathrm{~V}$ and $+8 \mathrm{~V}$ ), as also shown in the upper part of Fig. $2 \mathrm{a}$. By suitable measurements of the currents $\left(I_{\mathrm{T}}\right)$ and the measured binding energy positions, the values for all of the circuit elements can be derived, with the exception of the capacitance of the oxide layer $\left(C_{\mathrm{ox}}\right)$. The time-dependent measurements are needed for this capacitance, as it was demonstrated in our previous work [21-23]. This procedure involves recording XPS data in different time-windows of $10 \mathrm{~ms}$ after pulsing of the external voltage source, as shown in Fig. 2b. The time constant can be obtained after extracting the binding energy positions with respect to time by fitting an exponential curve.

Measurements for the thick silicon oxide layer of $400 \mathrm{~nm}$ yield the corresponding equivalent circuit parameters given in Table 1, which are obtained from the PSpice Schematics program [24]. $R_{\mathrm{g}}$ is very small compared to $R_{\mathrm{s}}$, which indicates that the stray electron flow is negligible compared to the electron gun current flow. This means that the dominant mechanism of charge compensation is due to the electron gun current.

An important advantage of using the equivalent circuit model and simulation is the assessment of the factors contributing to the experimentally derived parameters. For example, the time-dependent measurements shown in Figs. $2 \mathrm{~b}$ and $3 \mathrm{~b}$ reveal a time constant $(\tau)$ of the entire system that is related to the capacitance of the oxide through

$\tau=C_{\mathrm{ox}} \times\left(R_{\mathrm{ox}} / / R_{\mathrm{s}} / / R_{\mathrm{g}}\right)$ 

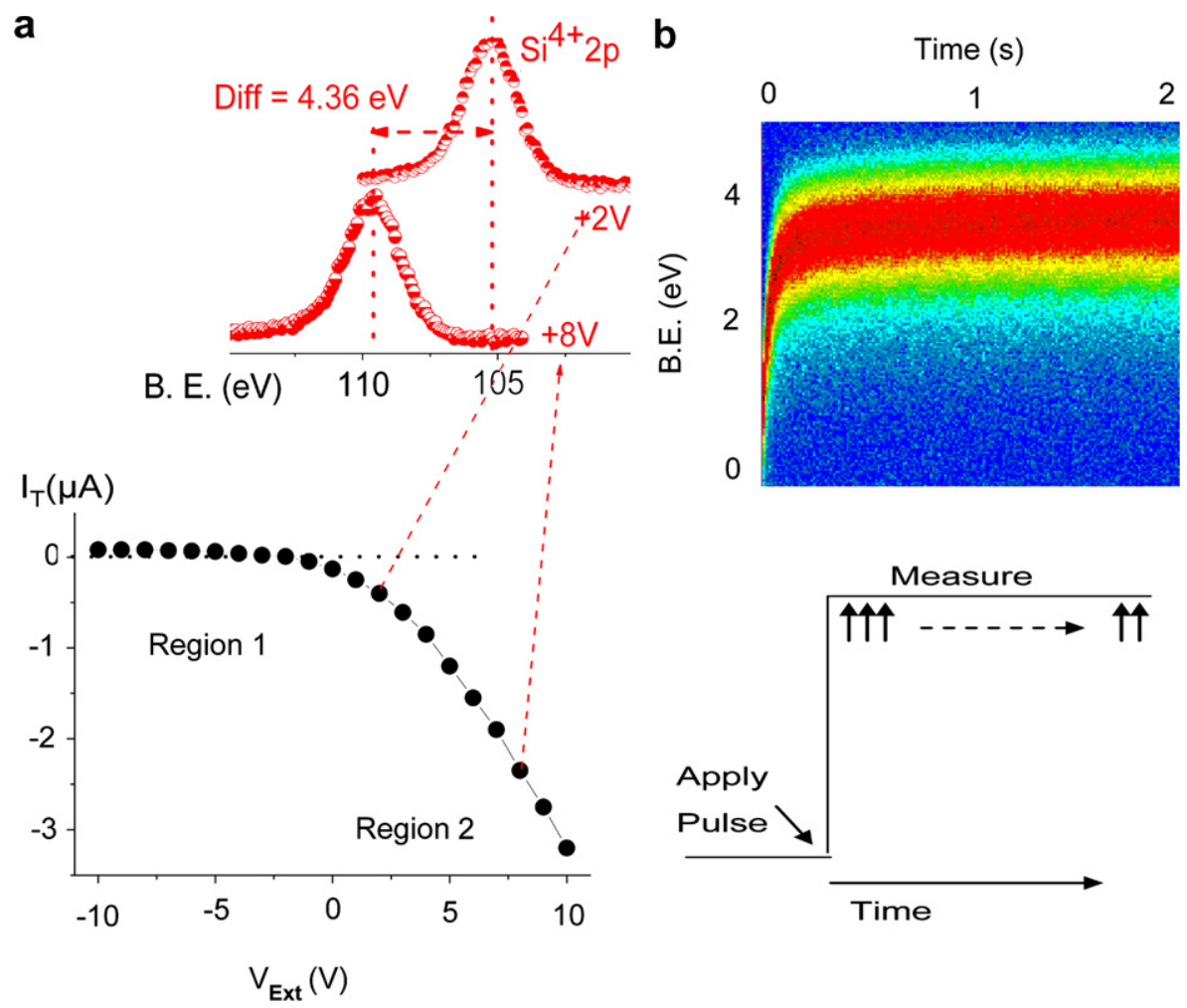

Fig. 2. (a) The measured total current flowing through the sample as a function of the external DC voltage and the Si2p peak recorded for $+2 \mathrm{~V}$ and $+8 \mathrm{~V}$, respectively. (b) The schematics of the pulsing procedure and the recorded 200 time-resolved spectra of the Si2p with $10 \mathrm{~ms}$ time-windows.

Table 1

Equivalent circuit parameters found for a thick silicon oxide layer of $400 \mathrm{~nm}$

\begin{tabular}{lllllll}
\hline$R_{\text {ox }}(\mathrm{M} \Omega)$ & $C_{\text {ox }}(\mathrm{nF})$ & $R_{\mathrm{s}}(\mathrm{M} \Omega)$ & $V_{\mathrm{s}}(\mathrm{V})$ & $R_{\mathrm{g}}(\mathrm{M} \Omega)$ & $V_{\mathrm{g}}(\mathrm{V})$ & $I_{\mathrm{s}}(\mathrm{nA})$ \\
\hline 1.4 & 140 & 135 & -5.25 & 1.5 & 1.39 & 40 \\
\hline
\end{tabular}

a

Exp. $\left(\mathrm{I}_{\mathrm{T}}\right)$

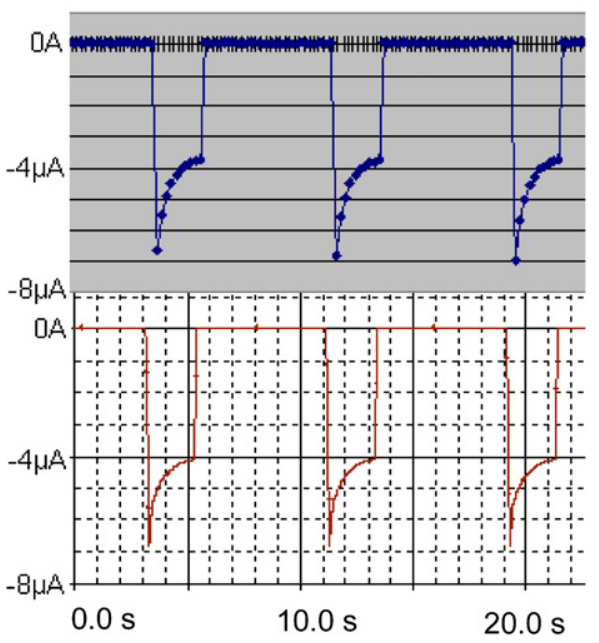

$\operatorname{Sim} .\left(\mathrm{I}_{\mathrm{T}}\right)$
Hence, not only the resistance of the oxide, but also all other resistances have to be taken into account to obtain a meaningful capacitance value.

After determining the equivalent circuit parameters involved, several simulations have been carried out and compared with measurements during data recording, in which

b
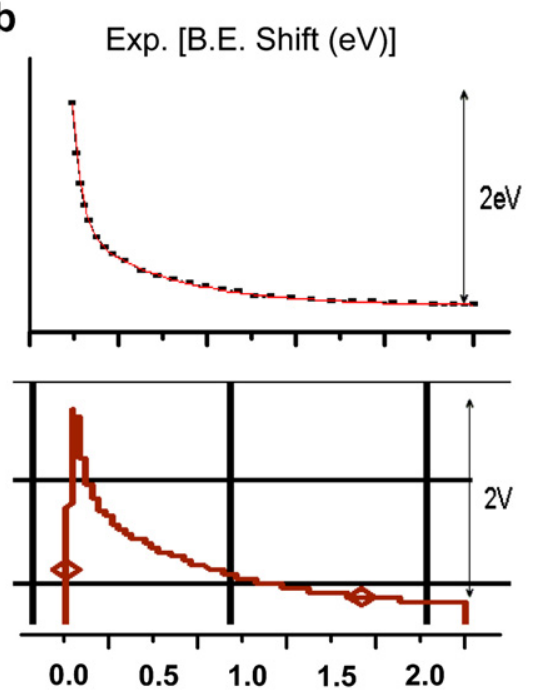

Sim. $\left(\mathrm{V}_{\mathrm{ox}}\right)$

Fig. 3. (a) The measured and simulated current passing through the sample, after application of a square pulse going from $-10 \mathrm{~V}$ to $+10 \mathrm{~V}$. (b) The measured B.E. shift of the Si2p peak as a function of time and the simulated $V_{\text {ox }}$. 
an input voltage pulse changing from $-10 \mathrm{~V}$ to $10 \mathrm{~V}$ was applied. In Fig. 3a, the total current measurements and the result of the simulation are shown. In Fig. 3b, the measured shift in the position of the Si2p peak is compared with the binding energy spectra of the oxide $\left(V_{\text {ox }}\right)$, using the values obtained from Table 1 . As it can easily be gathered from these figures, the agreement between simulations and measured parameters is remarkable. Our measurements have also been extended to cases where an RC network circuit was purposely added externally to the system to yield a perfect match between the measurements and simulations.

The $R_{\mathrm{ox}}$ and $C_{\mathrm{ox}}$ values derived from our measurements and those derived from the known geometry of the oxide layer $(400 \mathrm{~nm} \times 4 \mathrm{~mm} \times 8 \mathrm{~mm})$ should not be compared, because the XPS derived values are related with trapping and detrapping of the holes in the valence band of the oxide under X-ray and low-energy electron exposure. A similar XPS derived measurement was recently reported by Cohen as $0.83 \mathrm{M} \Omega / \mathrm{nm}$ for the silicon oxide layer which is in the same order of magnitude as ours [12].

One of the important applications of the equivalent circuit simulations can be the prediction of the zero surface potential condition, i.e. to estimate the case where measurements are carried out in the truly neutralized state of the sample. Accordingly, the zero surface potential is defined as the point where the applied voltage is cancelled by the IR drop at the surface

$V_{\text {ox }}\left(V_{\text {ext }}\right)=V_{\text {ext }}-R_{\text {ox }} \cdot I_{\mathrm{T}}\left(V_{\text {ext }}\right)=0$

This condition is fulfilled when an external voltage of $+0.65 \mathrm{~V}$ is applied to our sample.

Equivalent circuit simulations can also be used to accurately estimate the effects of changing various types of experimental parameters on the surface potential. For instance, it was predicted by Cazaux that doubling the Xray flux would not necessarily double the surface potential $[10,11]$. This was easily checked by our simulation by doubling $I_{\mathrm{p}}$, and the result was as estimated; the corresponding recorded shift was less than double.

\section{Conclusions}

In summary, by using a readily available PSpice simulation program, it is possible to derive information related to electrical parameters of surface structures and/or the XPS spectrometers in use. In this contribution, a simple Si oxide system for demonstrating the feasibility of the simulations was chosen. However, it is obvious that it can easily be extended to more complex and heterogeneous surface struc- tures. It is also possible to extend these measurements/ simulations for accurate estimations of the surface potential(s) for various types of stimuli (sinusoidal, square-wave, saw-tooth, etc.) for extracting time-dependent and/or transient response. This can also be applied to estimate the effects of different experimental conditions like X-ray flux, presence or absence of electron gun, etc. Finally, this electrical modeling approach can be adopted to other surface analysis techniques like AES or SEM.

\section{Acknowledgements}

This work was partially supported by TUBA (Turkish Academy of Sciences) and TUBITAK (The Scientific and Technological Research Council of Turkey) through the Grant No. 106T409.

\section{References}

[1] D. Briggs, M.P. Seah, Practical Surface Analysis, second ed., vol. 1, Wiley, Chichester, 1996.

[2] T.L. Barr, J. Vac. Sci. Technol. A 7 (1989) 1677.

[3] W.M. Lau, J. Appl. Phys. 65 (1989) 2047.

[4] W.M. Lau, X.W. Wu, Surf. Sci. 245 (1991) 345.

[5] B.J. Tielsch, J.E. Fulgum, Surf. Interface Anal. 24 (1996) 422; B.J. Tielsch, J.E. Fulgum, Surf. Interface Anal. 24 (1996) 459; B.J. Tielsch, J.E. Fulgum, Surf. Interface Anal. 25 (1997) 904.

[6] I. Doron-Mor, A. Hatzor, A. Vaskevich, T. van der Boom-Moav, A. Shanzer, I. Rubinstein, S.R. Cohen, H. Cohen, Nature (London) 406 (2000) 382.

[7] K. Shabtai, I. Rubinstein, S.R. Cohen, H. Cohen, J. Am. Chem. Soc. 122 (2000) 4959.

[8] J.P. Countour, G. Mouvier, Chem. Phys. Lett. 33 (1975) 237.

[9] W.M. Lau, Appl. Phys. Lett. 54 (1989) 338.

[10] J. Cazaux, J. Electron Spectosc. Relat. Phenom. 105 (1999) 155.

[11] J. Cazaux, J. Electron Spectosc. Relat. Phenom. 113 (2000) 15.

[12] H. Cohen, Appl. Phys. Lett. 85 (2004) 1271.

[13] H. Cohen, O.V. Zenkina, A.D. Shukla, M.E. Van der Boom, J. Phys. Chem. B 110 (2006) 1506.

[14] H. Cohen, S.K. Sarkar, G. Hodes, J. Phys. Chem. B 110 (2006) 25508.

[15] H. Cohen, R. Maoz, J. Sagiv, Nano Lett. 11 (2006) 2462.

[16] B. Ulgut, S. Suzer, J. Phys. Chem. B 107 (2003) 2939.

[17] F. Karadas, G. Ertas, S. Suzer, J. Phys. Chem. B 108 (2004) 1515.

[18] S. Suzer, A. Dana, J. Phys. Chem. B 110 (2006) 19112.

[19] A. Dana, Macromolecules 40 (2007) 4109.

[20] D.F. Mitchell, K.B. Clark, J.A. Bardwell, W.N. Lennard, G.R. Massoumi, I.V. Mitchell, Surf. Interface Anal. 21 (1994) 44.

[21] U.K. Demirok, G. Ertas, S. Suzer, J. Phys. Chem. B 108 (2004) 5179.

[22] G. Ertas, U.K. Demirok, A. Atalar, S. Suzer, Appl. Phys. Lett. 86 (2005) 183110.

[23] E. Karabudak, U.K. Demirok, S. Suzer, Surf. Sci. Lett. 600 (2006) L12.

[24] PSpice Schematics, Version 9.1, Cadence Design Systems. <http:// www.cadence.com $>$. 